\title{
Copy Number Variation of the Salivary Amylase Gene and Glucose Metabolism in Healthy Young Japanese Women
}

\author{
Ryoko Higuchi ${ }^{\text {a }}$, Taizo Iwane ${ }^{\mathrm{a}}$, Ayaka Iida ${ }^{\mathrm{a}}$, Kei Nakajima ${ }^{\mathrm{a}, \mathrm{b}, \mathrm{c}, \mathrm{d}}$
}

\begin{abstract}
Background: Many studies have shown that low copy number variation $(\mathrm{CNV})$ of the salivary amylase gene $(A M Y 1)$ and low serum amylase concentration are associated with impaired glucose metabolism and obesity. We aimed to clarify the conflicting results of previous studies by examining $A M Y 1$ expression and metabolic indices in a homogenous group of healthy participants.
\end{abstract}

Methods: Sixty healthy non-obese young Japanese women aged 20 - 39 years were examined for $A M Y 1 \mathrm{CNV}$, salivary amylase, body mass index (BMI) and serum parameters including glycated hemoglobin (HbA1c), ketones, and total, salivary and pancreatic amylase. Respiratory quotient at rest and changes in blood glucose after starch loading were also examined.

Results: $A M Y 1 \mathrm{CNV}$ (range, 4 - 14) and the level of serum salivary amylase were correlated inversely with $\mathrm{HbA1c}(\mathrm{r}=-0.36, \mathrm{P}=0.003$ and $r=-0.30, P=0.02$, respectively), whereas the percentage of serum salivary amylase in total serum amylase was positively correlated with blood glucose at 30 and $45 \mathrm{~min}$ after starch loading $(\mathrm{r}=0.38, \mathrm{P}$ $=0.004$ and $\mathrm{r}=0.27, \mathrm{P}=0.04$, respectively). The level of serum total amylase, but not $A M Y 1 \mathrm{CNV}$, was correlated inversely with $\mathrm{BMI}(\mathrm{r}=$ $-0.29, \mathrm{P}=0.02)$. Logistic regression analysis showed that low $A M Y 1$ CNV (4 - 7) was significantly associated with an HbA1c of $\geq 5.4 \%$ ( $34 \mathrm{mmol} / \mathrm{mol}$ ) even after adjustment for age, BMI and energy consumption, compared with high $A M Y 1 \mathrm{CNV}(8$ - 14).

Conclusions: Although a higher percentage of serum salivary amylase was associated with higher levels of blood glucose at the early stage after starch loading, low $A M Y 1 \mathrm{CNV}$ was associated with

Manuscript submitted December 30, 2019, accepted February 21, 2020

aSchool of Nutrition and Dietetics, Faculty of Health and Social Services, Kanagawa University of Human Services, 1-10-1 Heisei-cho, Yokosuka, Kanagawa 238-8522, Japan

bDepartment of Endocrinology and Diabetes, Saitama Medical Center, Saitama Medical University, 1981 Kamoda, Kawagoe, Saitama 350-8550, Japan ${ }^{\mathrm{c}}$ Graduate School of Health Innovation, Kanagawa University of Human Services, Research Gate Building Tonomachi 2-A, 3-25-10 Tonomachi, Kawasaki, Kanagawa 210-0821, Japan

${ }^{\mathrm{d} C}$ Corresponding Author: Kei Najajima, School of Nutrition and Dietetics, Faculty of Health and Social Services, Kanagawa University of Human Services, 1-10-1 Heisei-cho, Yokosuka, Kanagawa 238-8522, Japan.

Email: nakajima-rsh@kuhs.ac.jp

doi: https://doi.org/10.14740/jocmr4082 chronic unfavorable glucose metabolism in healthy non-obese young women in Japan.

Keywords: Amylase gene; Salivary amylase; BMI; HbA1c; Serum amylase; Starch loading

\section{Introduction}

In recent decades, several studies have shown that low copy number variation $(\mathrm{CNV})$ of the salivary amylase gene (AMYI) and low serum amylase concentration are associated with obesity and impaired glucose metabolism [1-10]. However, the results of these studies are conflicting, because some studies have shown no association of $A M Y 1 \mathrm{CNV}$ with obesity and impaired glucose metabolism [11-16]. These conflicting results may be due to the heterogeneity in patient samples in terms of racial and genetic background, various complications and acquired physical conditions, and the use of different study methods particularly for the assessment of AMY1 CNV [17].

Although most of the previous studies have focused on $A M Y 1 \mathrm{CNV}$ and obesity/diabetes [1-10], few studies have considered $A M Y 1$ expression in saliva, which is reflected in measurements of oral and serum salivary amylase, and is a main contributor to total serum amylase. Furthermore, serum amylase, which comprises the salivary and pancreatic isoforms of amylase, has rarely been considered in the interplay of $A M Y 1$ CNV, obesity and glucose metabolism.

We aimed, therefore, to investigate the fundamental relationships of $A M Y 1 \mathrm{CNV}$, salivary amylase and serum salivary/ pancreatic amylase with metabolic indices including body mass index (BMI) in a homogenous sample of healthy nonobese young women.

\section{Materials and Methods}

\section{Design and participants}

We recruited 60 apparently healthy female non-smokers of Japanese origin, aged 20 - 39 years, with normal BMI $(<25.0$ $\mathrm{kg} / \mathrm{m}^{2}$ ) and no history of metabolic disorders. This study was approved by the Ethics Committee of Kanagawa University of Human Services (ID number 71-31). 
Table 1. Characteristics of the Study Participants

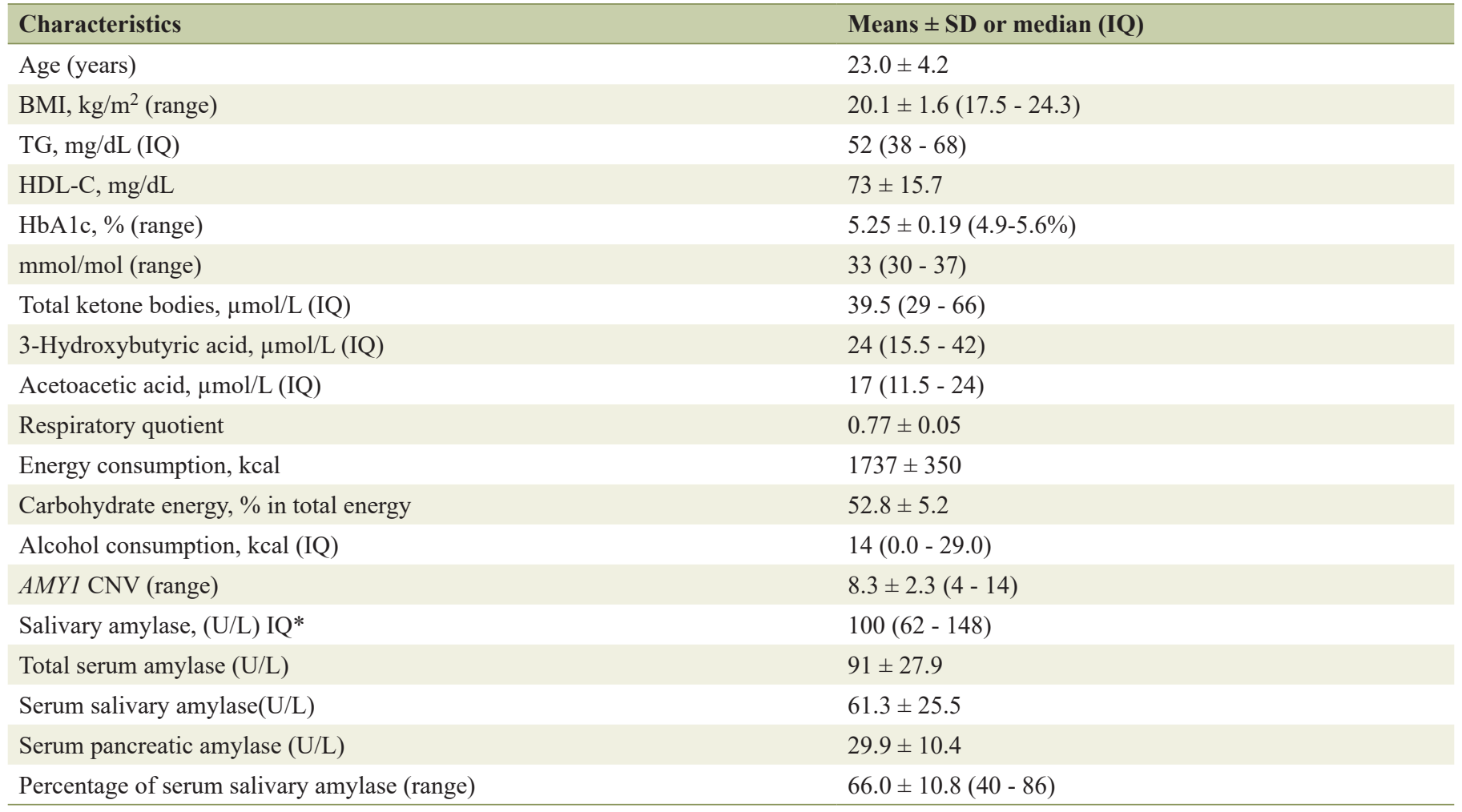

*Divided by the protein concentration in each saliva sample. SD: standard deviation; BMI: body mass index; HDL-C: high-density lipoprotein cholesterol; HbA1c: glycated hemoglobin; AMY1: salivary amylase gene; CNV: copy number variation; IQ: interquartile range; TG: triglycerides; FPG: fasting plasma glucose.

\section{Measurements}

Laboratory measurements were performed in the morning following an overnight fast. Biochemical measurements including glycated hemoglobin ( $\mathrm{HbAlc})$, ketones and amylase (total serum, salivary and pancreatic) were performed using standard automated methods. Blood glucose concentration after starch loading with a 130-g rice ball was measured using a Glutest-Neo alpha glucose meter (Sanwa-Kagaku-Kenkyusho, Nagoya, Japan). We calculated areas under the blood glucose based on the trapezoid rule. $A M Y 1$ expression was assessed by Droplet Digital PCR, which is preferred for its precise measurement [5, 17]. Briefly, TaqMan Copy Number Assays for AMY1A (AMY1A:Hs07226362_cn) and restriction enzyme DraI (Anza 77 DraI; both from Thermo Fisher Scientific) were used. The concentrations of amylase and total protein in saliva samples were measured by a kinetic reaction assay (Salivary alpha-Amylase Assay Kit, Salimetrics, Carlsbad, CA, USA) and a bicinchoninic acid assay protein assay (Pierce BCA Protein Assay Kit, Thermo Fisher Scientific), respectively. To estimate the respiratory quotient (RQ), respiratory monitoring for $5 \mathrm{~min}$ at rest was conducted after the adequate stabilization using an AR-1 portable gas monitor (ARCO SYSTEM Inc., Japan). To estimate an individual's consumption of energy, protein, lipids and carbohydrate, a food frequency questionnaire based on food groups [18] was used.

\section{Statistical analysis}

Data are expressed as the mean \pm standard deviation (SD) or median (interquartile range). Difference in areas under the blood glucose between low (range, 4 - 7) and high $A M Y 1 \mathrm{CNV}$ (range, 8 - 14) was tested by Mann-Whitney U test. Correlations between variables were tested by Spearman's correlation because of the high likelihood of non-parametric distributions in the parameters. Logistic regression analysis was used to test the association between low $A M Y 1 \mathrm{CNV}$ (range, 4 - 7) and $\mathrm{HbAlc} \geq 5.4 \%(34 \mathrm{mmol} / \mathrm{mol})$, a criteria for gestational diabetes $[19,20]$, compared with high $A M Y 1$ CNV (range, 8 14), considering potential confounders including age, BMI and energy consumption. Statistical analysis was performed using SAS Enterprise Guide (SAS-EG 7.1) in SAS version 9.4 (SAS Institute, Cary, NC, USA). A P value $<0.05$ was considered to indicate statistical significance.

\section{Results}

The characteristics of the participants are shown in Table 1; all of the parameters were within normal ranges. The percentage of salivary amylase in total serum amylase was $66.0 \pm 10.8 \%$. Figure 1 shows the levels of blood glucose after starch loading according to high (8 - 14) and low AMY1 CNV (4 - 7). Al- 


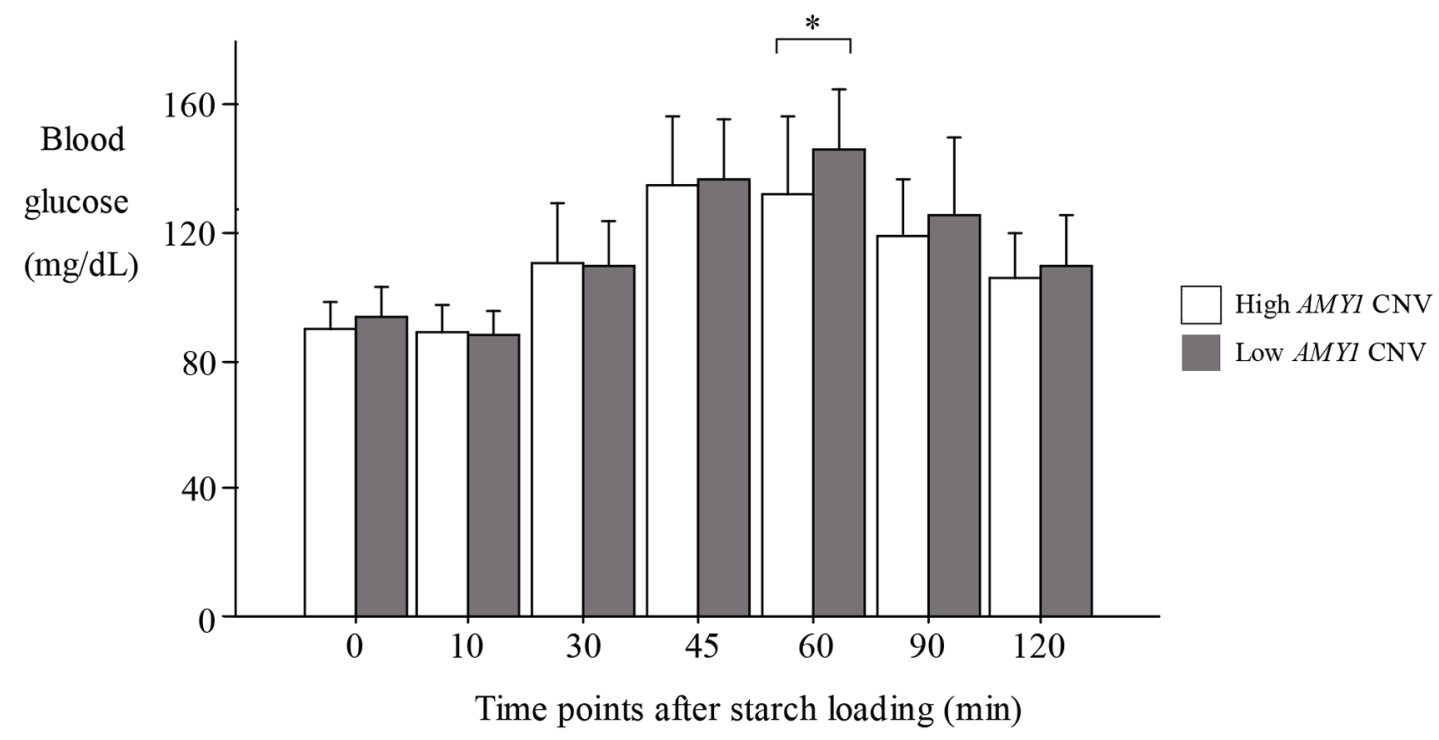

Figure 1. Levels of blood glucose after starch loading. Data are expressed as the mean $\pm \mathrm{SD}$. ${ }^{*} \mathrm{P}<0.05$, $t$-test, high vs. low AMY1 CNV. High AMY1 CNV: 8 - 14; low AMY1 CNV: 4 - 7. SD: standard deviation; CNV: copy number variation; AMY1: salivary amylase gene.

though significant difference was observed at 60 min between two groups, no difference was observed at other time points. Figure 2 shows the changes of blood glucose from baseline. No significant difference was observed at any time points between two groups. Simultaneously, we found no significant difference in the area under the blood glucose between two groups (medians (interquartile ranges), 3,102 (2,347 - 3,710) for high $A M Y 1 \mathrm{CNV}$ and 3,305 (2,288 - 4,113) for low AMY1
$\mathrm{CNV}, \mathrm{P}=0.50$, data are not shown).

Table 2 shows the results of correlation analysis among the parameters investigated. $A M Y 1 \mathrm{CNV}$ (range, 4 - 14) and serum salivary amylase were inversely correlated with $\mathrm{HbA} 1 \mathrm{c}(\mathrm{r}=$ $-0.36, \mathrm{P}=0.003$ and $\mathrm{r}=-0.30, \mathrm{P}=0.02$, respectively), whereas serum salivary amylase was positively correlated with blood glucose at $30 \mathrm{~min}$ after starch loading $(\mathrm{r}=0.26, \mathrm{P}=0.04)$. The percentage of serum salivary amylase was also positively

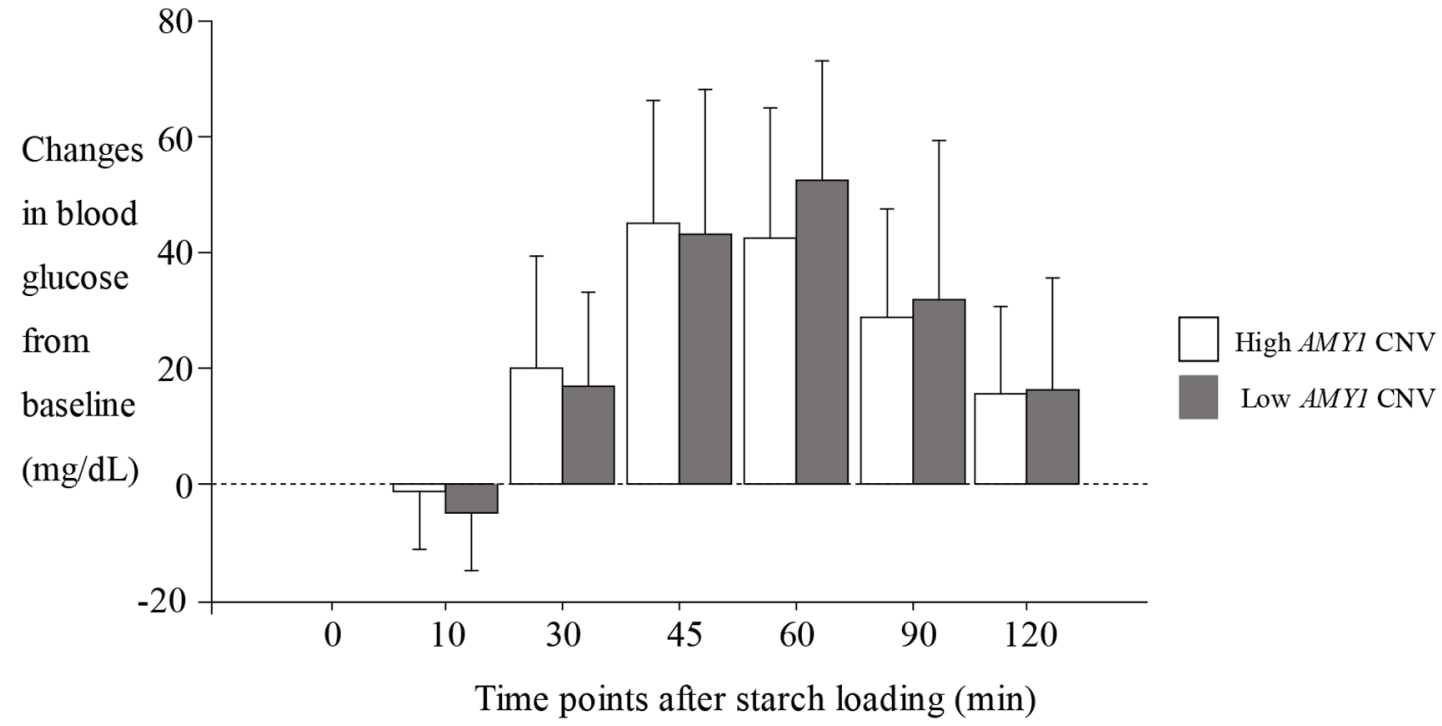

Figure 2. Changes of blood glucose from baseline after starch loading. Data are expressed as the mean \pm SD. High $A M Y 1 C N V$ : 8 - 14; low AMY1 CNV: 4 - 7. SD: standard deviation; CNV: copy number variation; AMY1: salivary amylase gene. 
Table 2. Correlation Coefficients Between the Study Parameters

\begin{tabular}{|c|c|c|c|c|c|c|c|}
\hline & AMV1 CNV & Solivary amylase & & Ser & m amylase & & $\mathbf{R O}$ \\
\hline & ANMY CN & Sainvary amyiase & Total & Salivary & Pancreatic & $\%$ Salivary & NQ \\
\hline BMI & -0.02 & 0.02 & $-0.29 *$ & -0.22 & -0.18 & 0.01 & 0.09 \\
\hline Triglyceride & -0.15 & 0.23 & -0.04 & -0.05 & -0.01 & -0.04 & 0.10 \\
\hline HDL-C & -0.14 & -0.17 & -0.16 & -0.20 & 0.06 & -0.17 & 0.02 \\
\hline Acetoacetic acid & 0.11 & -0.01 & -0.16 & -0.13 & -0.12 & 0.06 & $-0.36^{* *}$ \\
\hline 3-Hydroxybutyric acid & 0.17 & -0.06 & -0.09 & -0.05 & -0.15 & 0.14 & $-0.45 * * *$ \\
\hline$A M Y 1 \mathrm{CNV}$ & - & - & - & - & - & - & - \\
\hline Salivary amylase & $0.39^{* *}$ & - & - & - & - & - & - \\
\hline Pancreatic & -0.08 & 0.13 & $0.40 * *$ & 0.04 & - & - & - \\
\hline$\%$ Salivary & $0.37^{* *}$ & 0.24 & $0.36^{* *}$ & $0.68 * * *$ & $-0.64 * * *$ & - & - \\
\hline RQ & -0.06 & -0.02 & 0.21 & 0.12 & $0.29 *$ & -0.13 & - \\
\hline Blood glucose time cour & & & & & & & \\
\hline Baseline & -0.14 & -0.12 & -0.11 & -0.18 & 0.15 & -0.22 & 0.08 \\
\hline $10 \mathrm{~min}$ & -0.05 & 0.08 & -0.03 & 0.02 & -0.25 & 0.13 & 0.15 \\
\hline $30 \mathrm{~min}$ & 0.05 & 0.11 & 0.17 & $0.26^{*}$ & -0.18 & $0.38^{* *}$ & 0.11 \\
\hline $45 \min$ & -0.02 & -0.06 & 0.04 & 0.14 & -0.22 & $0.27^{*}$ & -0.07 \\
\hline $60 \mathrm{~min}$ & -0.14 & 0.04 & -0.20 & -0.10 & -0.22 & 0.16 & -0.18 \\
\hline
\end{tabular}

${ }^{*} \mathrm{P}<0.05 ;{ }^{*} \mathrm{P}<0.01 ;{ }^{* *} \mathrm{P}<0.001$. AMY1: salivary amylase gene; CNV: copy number variation; RQ: respiratory quotient; BMI: body mass index; HDL-C: high-density lipoprotein cholesterol; HbA1c: glycated hemoglobin.

correlated with blood glucose at 30 and $45 \min (\mathrm{r}=0.38, \mathrm{P}=$ 0.004 and $\mathrm{r}=0.27, \mathrm{P}=0.04$, respectively). The level of total serum amylase, but not $A M Y 1 \mathrm{CNV}$, was inversely correlated with $\mathrm{BMI}(\mathrm{r}=-0.29, \mathrm{P}=0.02)$. Only serum pancreatic amylase was positively correlated with RQ, which was significantly correlated with three indices related to serum ketones.
Logistic regression analysis showed that low $A M Y 1 \mathrm{CNV}$ (4 - 7) was significantly associated with HbAlc of $\geq 5.4 \%$, even after adjustment for age, BMI and energy consumption, compared with high $A M Y 1$ CNV $(8$ - 14) (Table 3). Furthermore, this association was strengthened by the further adjustment for salivary amylase adjusted for its concentration.

Table 3. Odds Ratios of Low AMY1 CNV for High HbA1c

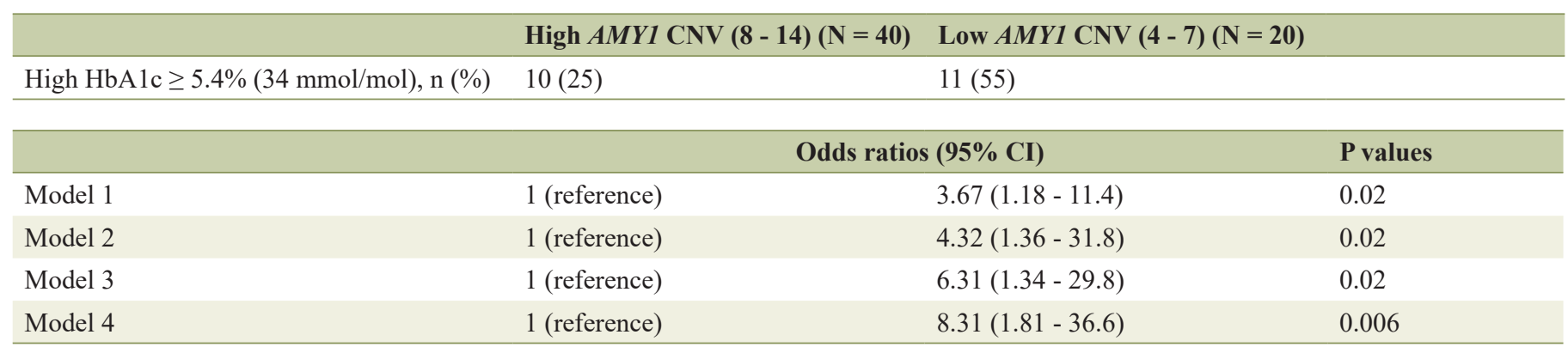

Model 1: unadjusted. Model 2: adjusted for age and BMI. Model 3: model 2 plus adjustments for energy consumption (kcal) and serum total amylase. Model 4: model 2 plus adjustments for energy consumption $(\mathrm{kcal})$ and salivary amylase for its concentration. AMY1: salivary amylase gene; CNV: copy number variation; Cl: confidence interval; HbA1c: glycated hemoglobin; BMI: body mass index. 


\section{Discussion}

Several studies published in the last decade have shown that low $A M Y 1 \mathrm{CNV}$ is associated with obesity [1-10]. In this study, however, no significant direct association between $A M Y 1 \mathrm{CNV}$ and BMI was observed. This result may have been influenced by our selection of non-obese female participants because the common association between BMI and $\mathrm{HbAlc}$ was also not observed $(\mathrm{r}=-0.09, \mathrm{P}=0.48$, data not shown). By contrast, only the total serum amylase concentration was inversely associated with BMI, which is consistent with our previous study $[6,21]$. Therefore, roughly speaking, the relationship of $A M Y 1$ $\mathrm{CNV}$ with metabolic indices may differ from that of total serum amylase and salivary amylase.

High $A M Y 1 \mathrm{CNV}$ is generally considered to indicate a high level salivary amylase that takes advantage of a large amount of starch by digesting it into oligosaccharides. In consistent with this, high levels of serum salivary amylase and a high percentage of salivary amylase in total serum amylase were associated with high blood glucose levels at an early stage after starch loading in this study. This finding may be consistent with a study by Atkinson et al [13], but in contrast to a study by Mandel et al [1]. Nevertheless, AMY1 CNV and serum salivary amylase were inversely correlated with $\mathrm{HbA} 1 \mathrm{c}$, an index for glucose metabolism over a period of months. More specifically, low $A M Y 1 \mathrm{CNV}$ was associated with $\mathrm{HbA1c} \geq 5.4 \%$, independent of potential confounders. A plausible explanation for this is that healthy, non-obese individuals with high $A M Y 1$ $\mathrm{CNV}$ and serum salivary amylase represent the default condition, i.e. a readiness to absorb as many carbohydrates as possible by means of a high amount of salivary amylase in the oral cavity, which might be accelerated in those without impaired glucose metabolism, i.e. lower HbAlc. Until the current century, most of the human beings who ate grains lived under such conditions, wherein the supply of grains was an inadequate source of energy.

By contrast, when individuals living in modern times chronically consume large amounts of carbohydrate, which leads to high HbA1c, a high level of salivary amylase may be unnecessary to digest a large intake of starch, suggesting that the fundamental relationship between $A M Y 1 \mathrm{CNV}$ and glucose metabolism might have become modified.

Alternatively, healthy non-obese individuals with low AMY1 CNV who lack the perception for sweet taste may overeat, and an absent cephalic response to the early secretion of insulin may lead to a higher peak level of blood glucose after starch loading $[6,7]$. Both of these conditions predispose the individual to increasing risks for obesity and impaired glucose metabolism.

Furthermore, the inverse association between serum amylase and BMI may suggest a specific condition of "amylase resistance" in individuals with obesity and diabetes, who by definition also have insulin resistance. The action of insulin is crucial for the production of amylase in the pancreas, but probably also in the salivary glands $[22,23]$. Therefore, it is possible that insulin resistance leads to decreased secretion of amylase from the saliva and pancreas [6,7], and could be a physiological response in people with diabetes and obesity.
With regard to the correlations of serum pancreatic amylase with HbA1c, RQ and blood glucose after starch loading, it is noteworthy to consider that serum pancreatic amylase might have the opposite effect on glucose metabolism that serum salivary amylase has. This would strengthen the correlations between the percentage of serum salivary amylase and serum pancreatic amylase and blood glucose after starch loading. Therefore, the effect of salivary amylase on metabolic indices may be influenced by the level of pancreatic amylase.

Finally, in this study, all participants were non-obese young women without overt impaired glucose metabolism. Furthermore, the percentage of salivary amylase was higher $(66 \%)$ than previously considered (around 50\%) [6, 7]. Therefore, the current observations may not be applicable to other populations, such as men, elderly people and those with obesity, and as such caution should be exercised in the interpretation of the results.

In conclusion, although a higher percentage of serum salivary amylase was associated with higher levels of blood glucose early after starch loading, our finding that low $A M Y 1$ $\mathrm{CNV}$ was associated with chronic unfavorable glucose metabolism in a population of healthy non-obese young Japanese women deserves further study.

\section{Acknowledgments}

We thank Michelle Kahmeyer-Gabbe, PhD, from Edanz Group (www.edanz.com/ac) for editing a draft of this manuscript.

\section{Financial Disclosure}

This study was supported by grants from the Rice Stable Supply Support Organization (Public Interest Incorporated Association), Tokyo, Japan.

\section{Conflict of Interest}

The authors declare that there is no conflict of interest associated with this manuscript.

\section{Informed Consent}

Written informed consent was obtained from each participant.

\section{Author Contributions}

KN contributed to the overall study design. KN and RH contributed to the interpretation of the initial analysis and the discussion of the literature. RH, TI and AI measured parameters and collected the serum data and others including RQ, salivary amylase and their proteins. KN prepared the first draft of the manuscript, and all authors read and approved the manuscript. 


\section{Data Availability}

Any inquiries regarding supporting data availability of this study should be directed to the corresponding author.

\section{References}

1. Mandel AL, Breslin PA. High endogenous salivary amylase activity is associated with improved glycemic homeostasis following starch ingestion in adults. J Nutr. 2012;142(5):853-858.

2. Falchi M, El-Sayed Moustafa JS, Takousis P, Pesce F, Bonnefond A, Andersson-Assarsson JC, Sudmant PH, et al. Low copy number of the salivary amylase gene predisposes to obesity. Nat Genet. 2014;46(5):492-497.

3. Mejia-Benitez MA, Bonnefond A, Yengo L, et al. Beneficial effect of a high number of copies of salivary amylase AMY1 gene on obesity risk in Mexican children. Diabetologia. 2015;58:290-294.

4. Choi YJ, Nam YS, Yun JM, Park JH, Cho BL, Son HY, Kim JI, et al. Association between salivary amylase (AMY1) gene copy numbers and insulin resistance in asymptomatic Korean men. Diabet Med. 2015;32(12):1588-1595.

5. Peyrot des Gachons C, Breslin PA. Salivary amylase: digestion and metabolic syndrome. Curr Diab Rep. 2016;16(10):102.

6. Nakajima K. Low serum amylase and obesity, diabetes and metabolic syndrome: A novel interpretation. World J Diabetes. 2016;7(6):112-121.

7. Elder PJD, Ramsden DB, Burnett D, Weickert MO, Barber TM. Human amylase gene copy number variation as a determinant of metabolic state. Expert Rev Endocrinol Metab. 2018;13(4):193-205.

8. Pinho S, Padez C, Manco L. High AMY1 copy number protects against obesity in Portuguese young adults. Ann Hum Biol. 2018;45(5):435-439.

9. Leon-Mimila P, Villamil-Ramirez H, Lopez-Contreras BE, Moran-Ramos S, Macias-Kauffer LR, Acuna-Alonzo V, Del Rio-Navarro BE, et al. Low Salivary Amylase Gene (AMY1) copy number is associated with obesity and gut prevotella abundance in mexican children and adults. Nutrients. 2018;10(11):1607.

10. Venkatapoorna CMK, Ayine P, Parra EP, Koenigs T, Phillips M, Babu JR, Sandey M, et al. Association of Salivary Amylase (AMY1) gene copy number with obesity in alabama elementary school children. Nutrients. 2019;11(6):1379.

11. Tan VM, Ooi DS, Kapur J, Wu T, Chan YH, Henry CJ, Lee YS. The role of digestive factors in determining glycemic response in a multiethnic Asian population. Eur J Nutr. 2016;55(4):1573-1581.
12. Yong RY, Mustaffa SB, Wasan PS, Sheng L, Marshall CR, Scherer SW, Teo YY, et al. Complex copy number variation of AMY1 does not associate with obesity in two East Asian cohorts. Hum Mutat. 2016;37(7):669-678.

13. Atkinson FS, Hancock D, Petocz P, Brand-Miller JC. The physiologic and phenotypic significance of variation in human amylase gene copy number. Am J Clin Nutr. 2018;108(4):737-748.

14. Marquina C, Mousa A, Belski R, Banaharis H, Naderpoor $\mathrm{N}$, de Courten B. Increased Inflammation and Cardiometabolic Risk in Individuals with Low AMY1 Copy Numbers. J Clin Med. 2019;8(3):382.

15. Valsesia A, Kulkarni SS, Marquis J, Leone P, Mironova P, Walter O, Hjorth MF, et al. Salivary alpha-amylase copy number is not associated with weight trajectories and glycemic improvements following clinical weight loss: results from a 2-phase dietary intervention study. Am J Clin Nutr. 2019;109(4):1029-1037.

16. Shwan NAA, Armour JAL. No evidence for association of BMI with salivary amylase gene copy number in the UK 1958 birth cohort. Obesity (Silver Spring). 2019;27(9):1533-1538.

17. Ooi DS, Tan VM, Ong SG, Chan YH, Heng CK, Lee YS. Differences in AMY1 gene copy numbers derived from blood, buccal cells and saliva using quantitative and droplet digital PCR methods: flagging the pitfall. PLoS One. 2017;12(1):e0170767.

18. Takahashi K, Yoshimura Y, Kaimoto T, Kunii D, Komatsu T, Yamamoto S. Validation of a food frequency questionnaire based on food groups for estimating individual nutrient intake. Jpn J Nutr. 2001;59:221-232.

19. Soumya S, Rohilla M, Chopra S, Dutta S, Bhansali A, Parthan G, Dutta P. HbA1c: a useful screening test for gestational diabetes mellitus. Diabetes Technol Ther. 2015;17(12):899-904.

20. Inoue H, Ishikawa K, Takeda K, Kobayashi A, Kurita K, Kumagai J, Yokoh H, et al. Postpartum risk of diabetes and predictive factors for glucose intolerance in East Asian women with gestational diabetes. Diabetes Res Clin Pract. 2018;140:1-8.

21. Nakajima K, Oda E. Ketonuria may be associated with low serum amylase independent of body weight and glucose metabolism. Arch Physiol Biochem. 2017;123(5):293296.

22. Carter DA, Wobken JD, Dixit PK, Bauer GE. Immunoreactive insulin in rat salivary glands and its dependence on age and serum insulin levels. Proc Soc Exp Biol Med. 1995;209(3):245-250.

23. Rocha EM, Carvalho CR, Saad MJ, Velloso LA. The influence of ageing on the insulin signalling system in rat lacrimal and salivary glands. Acta Ophthalmol Scand. 2003;81(6):639-645. 